\title{
Real-world treatment patterns and patient- reported outcomes in episodic and chronic migraine in Japan: analysis of data from the Adelphi migraine disease specific programme
}

Kaname Ueda* ${ }^{*}$, Wenyu Ye ${ }^{2}$, Louise Lombard ${ }^{2}$, Atsushi Kuga', Yongin Kim², Sarah Cotton ${ }^{3}$, James Jackson ${ }^{3}$ and Tamas Treuer ${ }^{4}$

\begin{abstract}
Background: In Japan, detailed information on the characteristics, disease burden, and treatment patterns of people living with migraine is limited. The aim of this study was to compare clinical characteristics, disease burden, and treatment patterns in people with episodic migraine (EM) or chronic migraine (CM) using real-world data from clinical practice in Japan.

Methods: This was an analysis of data collected in 2014 by the Adelphi Migraine Disease Specific Programme, a cross-sectional survey of physicians and their consulting adult patients in Japan, using physician and patient questionnaires. We report patient demographics, prescribed treatment, work productivity, and quality-of-life data for people with CM ( $\geq 15$ headache days/month) or EM (not fulfilling CM criteria). In descriptive analyses, continuous and categorical measures were assessed using t-tests and Chi-squared tests, respectively.

Results: Physicians provided data for 977 patients (mean age 44.5 years; $77.2 \%$ female; $94.5 \%$ with EM, 5.5\% with CM). A total of 634/977 (64.9\%) invited patients (600 with EM; 34 with CM) also provided data. Acute therapy was currently being prescribed in $93.7 \%$ and $100 \%$ of patients with EM and CM, respectively $(p=0.069)$; corresponding percentages for current preventive therapy prescriptions were $40.5 \%$ and $68.5 \%(p<0.001)$. According to physicians who provided data, preventive therapy was used at least once by significantly fewer patients with EM than with CM $(42.3 \%$ vs. $68.5 \%$, respectively; $p<0.001)$. Among patients who provided physicians with information on issues with their current therapy (acute therapy: $n=668$ with EM, $n=38$ with CM; preventive therapy: $n=295$ with EM, $n=21$ with CM), lack of efficacy was the most frequently identified problem (acute therapy: EM 35.3\%, CM 39.5\% [p $=0$. 833]; preventive therapy: EM 35.3\%, CM 52.4\% [ $p=0.131]$ ). Moderate-to-severe headache-related disability (Migraine Disability Assessment total score $\geq 11)$ was reported by significantly fewer patients with EM than with CM (21.0\% vs. $60.0 \%$, respectively; $p<0.001$ ) among patients who provided data.

Conclusions: Preventive treatment patterns in people with EM versus CM differ in Japan, with both types of migraine posing notable disease burdens. Our findings demonstrate that more effective migraine therapies are required to reduce the burden of the disease.
\end{abstract}

Keywords: Chronic migraine, Episodic migraine, Migraine, Clinical practice, Treatment patterns, Patient-reported outcomes

\footnotetext{
* Correspondence: ueda_kaname@lilly.com

${ }^{1}$ Eli Lilly Japan K.K., 5-1-28, Isogamidori, chuo-ku, Kobe-shi 651-0086, Japan

Full list of author information is available at the end of the article
}

(c) The Author(s). 2019 Open Access This article is distributed under the terms of the Creative Commons Attribution 4.0 International License (http://creativecommons.org/licenses/by/4.0/), which permits unrestricted use, distribution, and reproduction in any medium, provided you give appropriate credit to the original author(s) and the source, provide a link to the Creative Commons license, and indicate if changes were made. 


\section{Background}

Migraine is a debilitating primary headache disorder, characterized by recurrent unilateral (one-sided) pulsatile headaches. According to the Headache Classification Committee of the International Headache Society (IHS), people with $\geq 15$ headache days per month (HDM) for more than 3 months, at least 8 of which meet criteria for a migraine attack and/or respond to acute migraine-specific medication, are classified as having chronic migraine (CM) [1]; those with $\leq 14 \mathrm{HDM}$ are classified as having episodic migraine (EM) [2].

The impact of migraine extends far beyond the physical pain of a migraine attack, with substantial effects on multiple aspects of an individual's life, including health-related quality of life (HRQoL) and day-to-day functioning [3, 4]. In 2016, the Global Burden of Diseases, Injuries, and Risk Factors study found migraine to be the second leading cause of years lived with disability (YLD) (after lower back pain), contributing 45.1 million of total YLDs globally [5].

Recent data indicate that migraine is highly prevalent worldwide: a systematic review and meta-analysis published in 2017 estimated the average global prevalence of migraine at the community level to be $11.6 \%(10.4 \%$ in Africa, 10.1\% in Asia, 11.4\% in Europe, 9.7\% in North America, and 16.4\% in Central and South America) [6].

The overall prevalence of migraine in Japan was reported to be $8.4 \%$ (6.6\% in men, $13.0 \%$ in women) in 1997 [7]). In 2004, the Daisen study, a population-based survey conducted in the Daisen area of the Tottori prefecture, reported a prevalence for migraine of $6.0 \%$ in residents aged 20 years or older. Migraine was more prevalent in women than in men $(9.1 \%$ vs. $2.3 \%$, respectively), with the highest rates among women aged 30-49 years [8]. Other surveys, conducted in Japanese high-school students, reported prevalences for migraine of $5.8 \%$ (5.5\% in boys, $6.1 \%$ in girls) in 2005 [9] and $4.8 \%$ (3.3\% in boys, $6.5 \%$ in girls) in 2007 [10].

Overall, most of the participants in these epidemiological surveys had not consulted medical facilities, despite migraine being the cause of notable disability in their everyday lives [8-10].

The Clinical Practice Guideline for Chronic Headache [11], published in 2013 by the Japanese Society of Neurology (JSN) and the Japanese Headache Society (JHS), serves as a helpful guide for healthcare professionals who treat people with migraine in Japan. However, only limited information is available on the characteristics, disease burden, and treatment patterns of people with EM or CM in Japan, in contrast to that reported for some other countries (e.g., the USA). The aim of the current study was to provide this information, using real-world data reported by people with EM or CM actively seeking treatment for their migraine in Japan and by their treating physicians, focusing on clinical characteristics (including comorbidities), acute and preventive treatments, concomitant medications, work productivity, and HRQoL. Additionally, the study sought to examine the burden of illness and disability across patient subgroups based on average monthly headache frequency.

\section{Methods}

\section{Study design and population}

This research was a retrospective analysis of cross-sectional survey data collected by the Japanese Adelphi Migraine Disease-Specific Programme (DSP) in 2014, which was conducted independently by Adelphi Real World (Bollington, UK). The Japanese Adelphi Migraine DSP surveyed physicians and their consulting adult patients with migraine and collected migraine-specific data pertaining to treatment practice, symptom prevalence, patient demographics, clinical outcomes, medication utilization, adherence patterns, healthcare utilization, work productivity, and HRQoL. The survey utilized the general methodology for Adelphi Migraine DSP surveys [12].

The study population comprised physicians (internists and neurologists) treating people with migraine and their migraine-diagnosed patients who were actively seeking care from their healthcare provider. The recruitment of physician and patient participants is described in Ford et al. [13]. Participating physicians were required to recruit ten patients who had a diagnosis of migraine. The first nine patients were to be consecutive, but to achieve a $10 \%$ oversampling of patients who had failed at least one prior preventive treatment, the tenth patient had to meet this requirement and may not have been consecutive.

Physicians were asked to complete a patient record form (PRF) for all patients (data from PRFs are referred to as physician-reported data throughout). The PRF asked detailed questions on patient demographics, diagnoses, severity of migraine, number of headache days, comorbidities, migraine diagnosis, monitoring, treatment history (acute and preventive), drivers of migraine therapy choice, adherence to therapy, and general patient management (including the frequency of consultations with the treating and other physicians).

Once they had completed each PRF, the physician then invited the patient to complete a confidential patient self-completion (PSC) form (data from PSC forms are referred to as patient-reported data throughout). The PSC form was designed to elicit greater detail on the patient's demographics, level of satisfaction with their migraine treatment, adherence to therapy, and health insurance status. The PSC form also assessed validated patient-reported outcome measures, including the $\mathrm{Mi}$ graine Disability Assessment (MIDAS) [14, 15], the EuroQol-5 Dimensions (EQ-5D) questionnaire [16], and 
the Work Productivity and Activity Impairment (WPAI) questionnaire [17]. The MIDAS was designed to quantify headache-related disability over a 3-month period $[14,15]$. The EQ-5D is a generic, multidimensional, HRQoL instrument that uses a health status profile and a visual analog scale (VAS) to rate global HRQoL [16]. The WPAI is a valid and reliable instrument for measuring work productivity, which captures missed work time, general health perceptions, physical and emotional role, pain, symptom severity, and work and usual activity impairment [17]. The WPAI in this study specifically captured the implications for work productivity and usual activity impairment due to headaches. Completion of the PSC form was at the discretion of the patient.

\section{Statistical methods}

Data were collected cross-sectionally (January to March 2014). After deidentification, data were quality checked, and final data were entered into an electronic database. These analyses used all available data from the 2014 Japan Adelphi Migraine DSP cross-sectional database for all patients who met the stated requirements for this study. On the basis of physician-reported data, patients were classified into two groups: EM (not fulfilling CM criteria) or CM ( $\geq 15$ HDM), in line with a previous Adelphi Migraine DSP survey [13]. Additionally, four subgroups were identified (EM 0-3, 4-7, and 8-14 HDM, and CM $\geq 15$ HDM) according to HDM frequency.

Descriptive statistical analyses were performed to compare clinical characteristics, disease burden, and treatment patterns between patients with EM and CM, and among the four subgroups. Two sample t-tests were used for continuous measures, and Fisher's exact (for small sample sizes) or Chi-squared tests were used for categorical measures when comparing patients with EM and CM.

Analysis of variance (ANOVA) was used to examine differences in mean disease disability, HRQoL, and work productivity scores across the four subgroups.

Summary statistical information was based on non-missing data. Statistical test results were conducted at a two-sided 5\% significance level. No adjustments were made for multiple comparisons. Analyses were conducted using SAS Enterprise Guide 7.12 (SAS Institute, Cary, NC, USA).

\section{Results}

A total of 114 physicians (64 internists, 50 neurologists) provided PRFs for 1019 patients with migraine, of which 977 were for adults and contained information on HDM. Of 977 invited patients, 634 (64.9\%; 600 with EM, 34 with $\mathrm{CM}$ ) also provided relevant data in PSC forms.

\section{Patient characteristics}

According to physician-reported data from 977 PRFs, the mean age of patients with migraine was 44.5 years; most had EM (94.5\%) and were female (77.2\%). The average number of migraine HDM was reported as 3.0 in patients with EM and 10.8 in those with $\mathrm{CM}(p<$ 0.001). The characteristics of patients with EM and CM were comparable $(p>0.05)$, with the exceptions that tension-type headache and medication-overuse headache $(\mathrm{MOH})$ were significantly more common in patients with CM than in those with EM $(p \leq 0.002)$ (Table 1$)$. Half (50.2\%) of patients with EM had 0-3 HDM, 29.2\% had 4-7 HDM, and 15.1\% had 8-14 HDM.

The most frequently physician-reported migraine-related symptoms considered most troublesome to patients (occurring in $\geq 5 \%$ of patients with $\mathrm{EM}$ or $\mathrm{CM}$ ) were pain related: unilateral pain (50.8\% of patients), pulsating/throbbing pain (20.8\%), bilateral pain (17.7\%), and pain worsened by activity $(8.5 \%)$ in patients with EM $(n=896)$, and unilateral pain $(37.7 \%)$, pulsating/ throbbing pain $(37.7 \%)$, bilateral pain $(24.5 \%)$, pain worsened by activity (7.5\%), nausea (7.5\%), and vomiting (7.5\%) in those with CM ( $n=53)$. Pulsating/throbbing pain was significantly $(p=0.004)$ more of a problem for those with CM than those with EM. Photophobia was reported by $1.5 \%$ and $3.8 \%$, and phonophobia was reported by $0.9 \%$ and $0 \%$, of patients with EM and CM, respectively.

Approximately half of all patients with EM $(n=455$ [49.3\%]) or CM $(n=29$ [53.7\%]) were reported by physicians as having comorbidities (Table 1). The most common comorbid conditions seen in these patients in both migraine groups ( $\geq 5 \%$ of those with EM or CM experiencing comorbidities) were hypertension $(27.5 \%$ and $27.6 \%$, respectively), hyperlipidemia (19.3\% and $20.7 \%)$, anxiety (18.0\% and $13.8 \%)$, sleep disorders $(12.7 \%$ and $17.2 \%)$, gastrointestinal disorders/dyspepsia $(13.6 \%$ and $24.1 \%)$, depression (11.2\% and $17.2 \%)$, obesity (8.8\% and $13.8 \%$ ), respiratory problems (asthma/chronic obstructive pulmonary disease/allergic rhinitis; $12.5 \%$ and $6.9 \%$ ), diabetes (6.4\% and 6.9\%), panic disorder (6.4\% and 3.4\%), and menstrual disorders (3.1\% and 6.9\%) (Additional file 2: Figure S1). There was no significant difference in the incidence of any comorbidity between patients with CM and EM. Concomitant medications were as expected for these comorbidities and are detailed in Additional file 1: Table S1.

\section{Treatment}

According to physician-reported data, over $85 \%$ of patients were consulting the physician about migraine during the visit in which they completed their PSC form (Table 2). Most patients with EM were consulting an internist (58.2\%), whereas a majority of those with $\mathrm{CM}$ were seeing a neurologist (74.1\%; Table 2). However, a greater proportion of patients with $\mathrm{CM}$ who were not currently receiving preventive therapy were consulting 
Table 1 Characteristics of patients with episodic migraine and chronic migraine: physician-reported data

\begin{tabular}{|c|c|c|c|c|}
\hline \multirow[t]{2}{*}{ Patient characteristic } & \multicolumn{2}{|l|}{ Cohort } & \multirow{2}{*}{$\begin{array}{l}\text { Total } \\
(N=977)\end{array}$} & \multirow[t]{2}{*}{$p$-value } \\
\hline & $\begin{array}{l}\text { Episodic migraine } \\
(N=923)\end{array}$ & $\begin{array}{l}\text { Chronic migraine } \\
(N=54)\end{array}$ & & \\
\hline Age (years), mean (SD) & $44.4(14.6)$ & $46.3(13.2)$ & $44.5(14.5)$ & $0.351^{\mathrm{a}}$ \\
\hline Female, n (\%) & $711(77.0)$ & $43(79.6)$ & $754(77.2)$ & $0.658^{b}$ \\
\hline Body mass index $\left(\mathrm{kg} / \mathrm{m}^{2}\right)$, mean $(\mathrm{SD})$ & $21.7(3.3)$ & $21.9(3.3)$ & $21.7(3.3)$ & $0.627^{\mathrm{a}}$ \\
\hline Smoking status, n (\%) & & & & $0.200^{b}$ \\
\hline Current smoker & $105(11.8)$ & $9(17.3)$ & $114(12.1)$ & \\
\hline Ex-smoker & $90(10.1)$ & $2(3.8)$ & $92(9.7)$ & \\
\hline Has never smoked & $698(78.2)$ & $41(78.8)$ & $739(78.2)$ & \\
\hline No. of migraine headache days (average per month), mean (SD) & $3.0(2.8)$ & $10.8(7.8)$ & $3.4(3.8)$ & $<0.001^{\mathrm{c}}$ \\
\hline Also had tension-type headaches, n (\%) & $194(21.0)$ & $21(38.9)$ & $215(22.0)$ & $0.002^{b}$ \\
\hline Also had medication-overuse headaches, n (\%) & $36(3.9)$ & $14(25.9)$ & $50(5.1)$ & $<0.001^{\circ}$ \\
\hline \multicolumn{5}{|l|}{ Home circumstances, n (\%) } \\
\hline Lives with spouse/partner & $515(57.7)$ & $34(66.7)$ & $549(58.2)$ & $0.205^{b}$ \\
\hline Lives with other family & $204(22.8)$ & $15(29.4)$ & $219(23.2)$ & $0.280^{\mathrm{b}}$ \\
\hline Lives with friends & $1(0.1)$ & 0 & $1(0.1)$ & $1.000^{c}$ \\
\hline Lives alone & $190(21.3)$ & $5(9.8)$ & $195(20.7)$ & $0.049^{b}$ \\
\hline Employment status, n (\%) & & & & $0.131^{c}$ \\
\hline Full-time & $433(48.0)$ & $23(45.1)$ & $456(47.8)$ & \\
\hline Part-time & $126(14.0)$ & $3(5.9)$ & $129(13.5)$ & \\
\hline Student & $35(3.9)$ & $1(2.0)$ & $36(3.8)$ & \\
\hline Unemployed & $104(11.5)$ & $8(15.7)$ & $112(11.8)$ & \\
\hline Retired & $12(1.3)$ & $3(5.9)$ & $15(1.6)$ & \\
\hline Homemaker & $187(20.7)$ & $13(25.5)$ & $200(21.0)$ & \\
\hline Other & $5(0.6)$ & 0 & $5(0.5)$ & \\
\hline Patient with any comorbidity, n (\%) & $455(49.3)$ & $29(53.7)$ & $484(49.5)$ & $0.529^{b}$ \\
\hline Total no. of comorbidities $(\max =37)^{\mathrm{d}}$, mean $(S D)$ & $1.8(1.2)$ & $2.1(1.8)$ & $1.8(1.3)$ & $0.378^{\mathrm{a}}$ \\
\hline
\end{tabular}

an internist (9/17 [52.9\%]) compared with $26 \%$ of the overall CM cohort. During the previous 12 months, similar mean numbers of internist/neurologist consultations for migraine were made by patients with EM (6.3 and 6.1, respectively) and CM (4.6 and 6.5, respectively), and migraine-related hospital visits were recorded for similar percentages of each group $(28.2 \%$ and $31.5 \%$, respectively) (Table 2).

Most patients with EM (93.7\%) or CM (100\%) were currently being prescribed an acute treatment for their migraine; $40.5 \%$ of those with EM and $68.5 \%$ of those with $\mathrm{CM}$ had current prescriptions for preventive therapy $(p<0.001)$ (Fig. 1). One-third of patients with EM (34.2\%) and two-thirds (68.5\%) of those with CM were being prescribed both acute and preventive therapy.
Only $9.0 \%$ and $8.5 \%$ of patients with EM or CM, respectively, were taking over-the-counter medications (mostly nonsteroidal anti-inflammatory drugs [NSAIDs] and acetaminophen/ethenzamide); the use of complementary therapies (most commonly massage, relaxation therapy, and Pilates/yoga) was $28.4 \%$ and $28.6 \%$ in patients with EM and CM, respectively.

In both patients with EM and those with CM who were receiving therapy, the majority of acute treatment prescriptions were for triptans and NSAIDs (Table 3). Current use of opioids as acute therapy was low $(0.2 \%$ of patients with EM; no patients with CM). Preventive therapy prescriptions most frequently comprised anticonvulsants and calcium antagonists (Table 3). Notable proportions of patients with EM or CM who were 
Table 2 Physician consultations and healthcare resource utilization: physician-reported data

\begin{tabular}{|c|c|c|c|c|}
\hline \multirow[t]{2}{*}{ Healthcare resource } & \multicolumn{2}{|l|}{ Cohort } & \multirow{2}{*}{$\begin{array}{l}\text { Total } \\
(n=977)\end{array}$} & \multirow[t]{2}{*}{$p$-value } \\
\hline & Episodic migraine $(n=923)$ & Chronic migraine $(n=54)$ & & \\
\hline Doctor specialty, n (\%) & & & & $<0.001^{2}$ \\
\hline Internist & $537(58.2)$ & $14(25.9)$ & $551(56.4)$ & \\
\hline Neurologist & $386(41.8)$ & $40(74.1)$ & $426(43.6)$ & \\
\hline Current consultation was for migraine, $\mathrm{n}(\%)$ & $794(86.1)$ & $51(94.4)$ & $845(86.6)$ & $0.081^{\mathrm{a}}$ \\
\hline $\begin{array}{l}\text { No. of times patient consulted for migraine in the } \\
\text { past } 12 \text { months - internist, mean (SD) }\end{array}$ & $6.3(5.4)$ & $4.6(4.9)$ & $6.2(5.4)$ & $0.193^{b}$ \\
\hline $\begin{array}{l}\text { No. of times patient consulted for migraine in the past } \\
12 \text { months - neurologist, mean (SD) }\end{array}$ & $6.1(4.7)$ & $6.5(5.7)$ & $6.1(4.8)$ & $0.576^{\mathrm{b}}$ \\
\hline $\begin{array}{l}\text { No. of patients making hospital visits for migraine in the } \\
\text { past } 12 \text { months, } \mathrm{n}(\%)\end{array}$ & $260(28.2)$ & $17(31.5)$ & $277(28.4)$ & $0.603^{\mathrm{a}}$ \\
\hline Ever had surgical intervention for migraine condition, $\mathrm{n}(\%)$ & $1(0.1)$ & 0 & $1(0.1)$ & $1.000^{c}$ \\
\hline
\end{tabular}

receiving preventive therapy were being prescribed the antidepressant/anxiolytic, amitriptyline, as the preventive therapy. Among patients with a current prescription for preventive therapy, the use of lomerizine, valproic acid, and amitriptyline was significantly higher in those with CM than in those with EM.

According to physician-reported data, acute therapy was used at least once (as first or higher line of therapy) by $95.2 \%$ and $100.0 \%$ of patients with EM or CM ( $p=$ 0.167 ), and preventive therapy was used at least once by
$42.3 \%$ and $68.5 \%$ of patients with EM or CM $(p<0.001)$ (Additional file 3: Figure S2).

Physicians reported that 51-60\% and 23-30\% (percentages based on total patient cohorts, although data were not available for all patients [either unknown or patients were not receiving therapy]) of patients with EM or CM indicated they were experiencing at least one issue with their current acute or preventive therapy, respectively (selected from a predetermined list of items in response to the question "Any issues with acute/

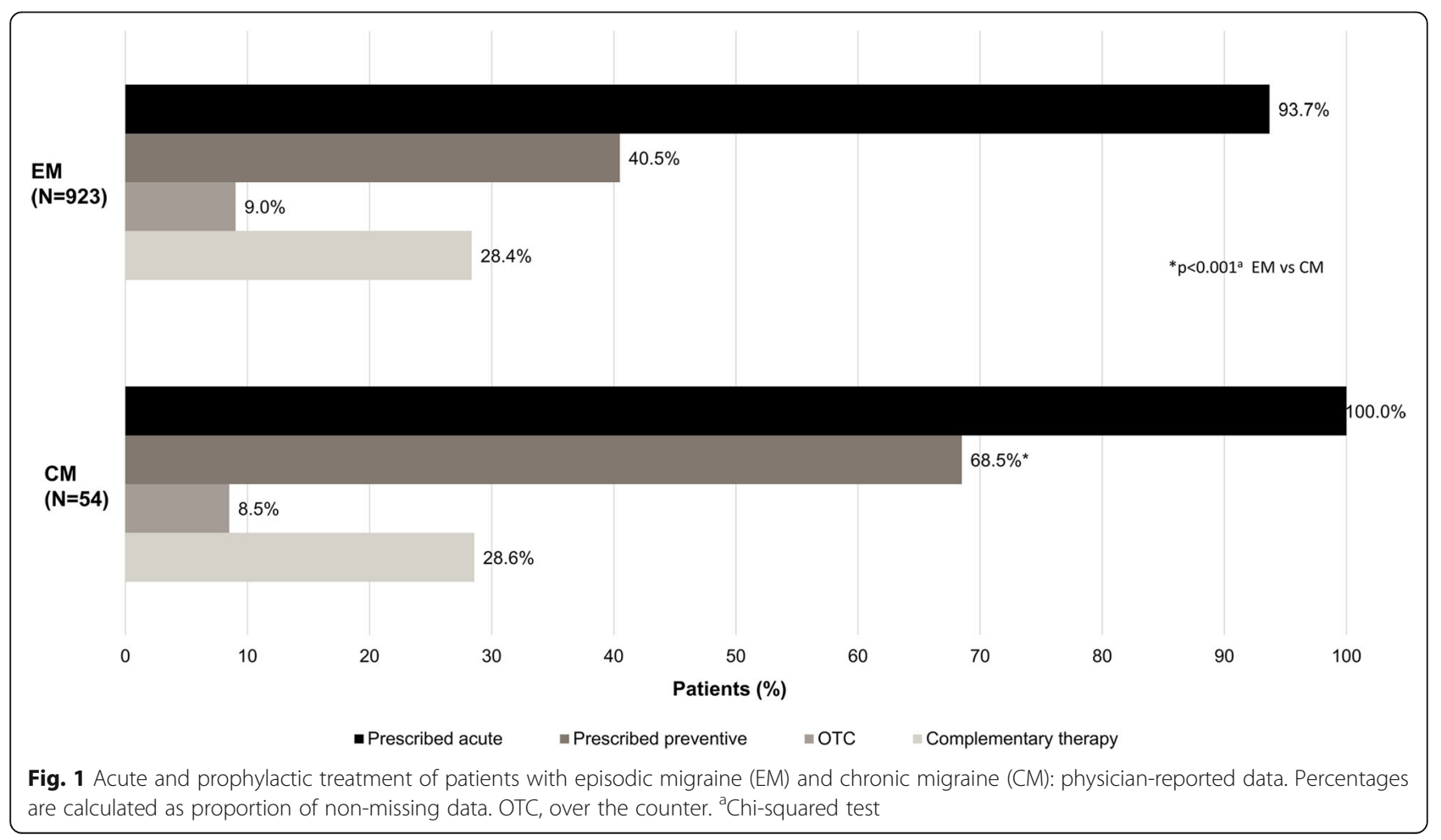


Table 3 Medications currently prescribed as acute or preventive migraine treatment for $\geq 5 \%$ of patients with episodic and chronic migraine: physician-reported data

\begin{tabular}{|c|c|c|c|c|}
\hline \multirow[t]{2}{*}{ Medication } & \multicolumn{2}{|l|}{ Cohort } & \multirow{2}{*}{$\begin{array}{l}\text { Total } \\
(n=977)\end{array}$} & \multirow[t]{2}{*}{$p$-value } \\
\hline & Episodic migraine $(n=923)$ & Chronic migraine $(n=54)$ & & \\
\hline \multicolumn{5}{|l|}{ Acute treatment } \\
\hline Eletriptan & $91(9.9)$ & $2(3.7)$ & $93(9.5)$ & $0.134^{\mathrm{a}}$ \\
\hline Naratriptan & $133(14.4)$ & $11(20.4)$ & $144(14.7)$ & $0.230^{\mathrm{a}}$ \\
\hline Sumatriptan & $162(17.6)$ & $7(13.0)$ & $169(17.3)$ & $0.386^{\mathrm{a}}$ \\
\hline Rizatriptan & $148(16.0)$ & $14(25.9)$ & $162(16.6)$ & $0.057^{\mathrm{a}}$ \\
\hline Zolmitriptan & $113(12.2)$ & $6(11.1)$ & $119(12.2)$ & $0.805^{\mathrm{a}}$ \\
\hline Loxoprofen & $275(29.8)$ & $18(33.3)$ & $293(30.0)$ & $0.581^{a}$ \\
\hline Diclofenac & $48(5.2)$ & $4(7.4)$ & $52(5.3)$ & $0.525^{\mathrm{b}}$ \\
\hline \multicolumn{5}{|c|}{ Preventive treatment } \\
\hline Lomerizine & $131(14.2)$ & $13(24.1)$ & $144(14.7)$ & $0.046^{\mathrm{a}}$ \\
\hline Valproic acid & $116(12.6)$ & $17(31.5)$ & $133(13.6)$ & $<0.001^{\mathrm{a}}$ \\
\hline Amitriptyline & $61(6.6)$ & $8(14.8)$ & $69(7.1)$ & $0.048^{b}$ \\
\hline
\end{tabular}

Data are $\mathrm{n}(\%)$

${ }^{a}$ Chi-squared test

${ }^{b}$ Fisher's exact test

preventive regimen?"). Among patients for whom PRF data on issues with acute therapy were available (668 with EM; 38 with CM), lack of efficacy was the most frequently experienced problem, reported for $35.3 \%$ and $39.5 \%$ of patients with EM or CM, respectively $(p=0.833)$ (Fig. 2a). Headache recurrence and $\mathrm{MOH}$ were the next most frequent problems with currently prescribed acute treatment in patients with CM; both were reported as a problem in significantly more patients with CM than with EM $(p<$ $0.05)$. Lack of efficacy was also the most frequently identified problem with current preventive therapy among patients for whom PRF data on issues with preventive therapy were available (295 with EM; 21 with CM), being reported for $35.3 \%$ of patients with EM and $52.4 \%$ of those

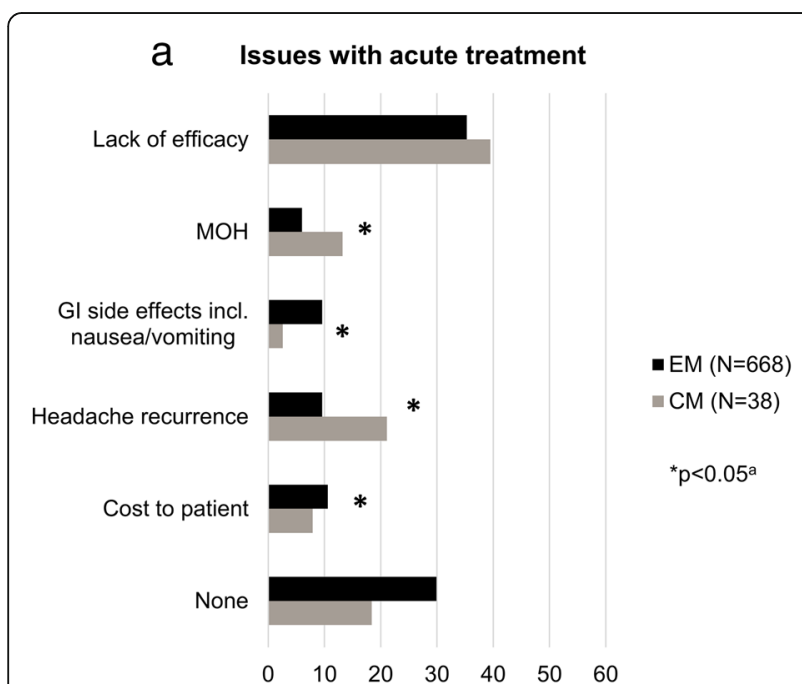

Patients responding to physician's question (\%)

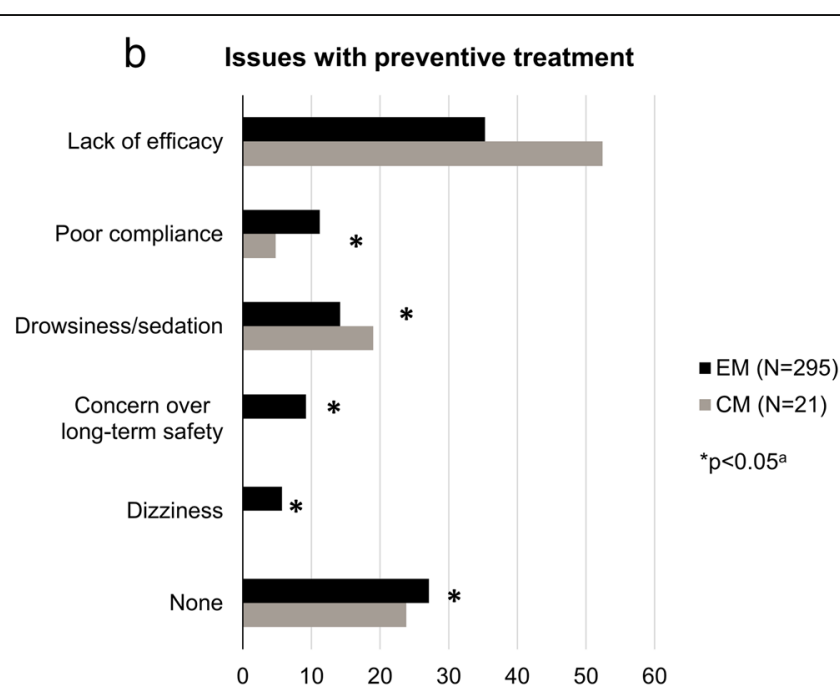

Patients responding to physician's question (\%)

Fig. 2 Issues with (a) acute and (b) prophylactic treatment reported for $>5 \%$ of patients with episodic migraine (EM) and chronic migraine (CM): physician-reported data. Issues were selected from a predetermined list by physicians when patients responded to the question "Any issues with acute/preventive regimen?". An item was checked only if considered an issue by the physician. Data were not available for all patients (either unknown or patient was not receiving treatment). Percentages are calculated as proportion of patients receiving acute therapy who reported at least one issue with therapy (acute treatment: $E M, N=668 ; C M, N=38$, total, $N=706$; preventive treatment: $E M, N=295 ; C M, N=21 ;$ total, $N=316$ ). "None" indicates the physician noted the patient had no acute/preventive treatment issues in the "other" category. Gl, gastrointestinal; $\mathrm{MOH}$, medication overuse headache. ${ }^{\mathrm{a}}$ Fisher's exact test 
with CM ( $p=0.131)$ (Fig. 2b). The next most frequent problems were drowsiness/sedation (reported as an issue in more patients with CM than with EM [p $<0.05])$. A numerically higher proportion of patients with EM than with $\mathrm{CM}$ for whom data on issues with therapy were reported indicated that they had no predetermined issues with their currently prescribed acute $(29.0 \%$ vs. $15.8 \%$, respectively, $p=0.209)$ or preventive $(26.8 \%$ vs. $23.8 \% ; p=0.529)$ treatment.

\section{Migraine burden}

Among patients who provided data in PSC forms (477 with EM; 20 with $\mathrm{CM}$ ), moderate-to-severe headache-related disability (MIDAS total score $\geq 11$ ) was reported by significantly fewer individuals with EM than with CM (21.0\% vs. $60.0 \% ; p<0.001)$. Mean MIDAS total score was lowest (4.8) in the EM 0-3 HDM subgroup but increased in the EM 4-7 HDM (11.0) and EM HDM 8-14 (15.7) subgroups, reaching 21.3 in the $\mathrm{CM} H \mathrm{HDM} \geq 15$ subgroup (higher scores signifying greater disability) $(p<0.001$ for trend) (Table 4). Over 70\% of patients with EM HDM 0-3 reported little or no disability (MIDAS total score $0-5$ ), and only $4.7 \%$ reported severe disability (MIDAS total score > 20 ); in contrast, $>30 \%$ of patients in the EM 8-14 HDM subgroup and those with $\mathrm{CM} \geq 15 \mathrm{HDM}$ had severe disability (Table 4).
EQ-5D utility and VAS scores were significantly ( $p \leq$ 0.005 ) lower (indicating poorer general health status and HRQoL, respectively) in patients with CM than in those with EM. EQ-5D utility scores were highest in the EM 0-3 HDM subgroup (0.89), decreasing over the HDM 4-7 (0.86) and 8-14 subgroups (0.82), and lowest in patients with $\mathrm{CM} \geq 15 \mathrm{HDM}$ (0.72; $p<0.001$ for trend) (Table 4). Similar findings were seen for HRQoL (EQ-5D VAS scores) (Table 4).

Patients with $\mathrm{CM}$ also experienced greater impairment and less productivity than those with EM, according to WPAI scores. Assessment of WPAI scores revealed that activity impairment increased with increasing numbers of HDM (from 0 to $3 \mathrm{HDM}$ to $\geq 15 \mathrm{HDM}$; $\mathrm{p}<0.001$ ), whereas numerical increases were seen for impairment at work and overall work impairment. Work time missed showed no increase across the four HDM subgroups (Table 4).

\section{Discussion}

The demographic profile of patients with EM and CM in Japan in this study differs in certain respects from that observed in large population-based studies in other countries $[2,4,18,19]$ and in another 2014 Adelphi Migraine DSP survey conducted in the USA [13].

The breakdown of patients classified as having EM (94.5\%) and CM (5.5\%) in the current study is consistent

Table 4 Burden of illness in patients with episodic migraine and chronic migraine: patient self-completion form data

\begin{tabular}{|c|c|c|c|c|c|c|}
\hline \multirow[t]{2}{*}{ Patient-reported outcomes } & \multicolumn{4}{|l|}{ Cohort } & \multirow{2}{*}{$\begin{array}{l}\text { Total } \\
(N=634)\end{array}$} & \multirow[t]{2}{*}{$p$-value } \\
\hline & $\begin{array}{l}\text { Episodic migraine } \\
0-3 \mathrm{HDM} \\
(n=331)\end{array}$ & $\begin{array}{l}\text { Episodic migraine } \\
4-7 \mathrm{HDM} \\
(n=183)\end{array}$ & $\begin{array}{l}\text { Episodic migraine } \\
8-14 \text { HDM } \\
(n=86)\end{array}$ & $\begin{array}{l}\text { Chronic migraine } \\
\geq 15 \text { HDM } \\
(n=34)\end{array}$ & & \\
\hline MIDAS total score, mean (SD) & $4.8(12.6)$ & $11.0(26.2)$ & $15.7(18.3)$ & $21.3(22.0)$ & $8.9(19.4)$ & $<0.001^{2}$ \\
\hline MIDAS disability category, n (\%) & & & & & & $<0.001^{b}$ \\
\hline Little/no disability & $191(74.9)$ & $86(57.7)$ & $29(39.7)$ & $6(30.0)$ & $312(62.8)$ & \\
\hline Mild disability & $32(12.5)$ & $25(16.8)$ & $14(19.2)$ & $2(10.0)$ & $73(14.7)$ & \\
\hline Moderate disability & $20(7.8)$ & $17(11.4)$ & $7(9.6)$ & $5(25.0)$ & $49(9.9)$ & \\
\hline Severe disability & $12(4.7)$ & $21(14.1)$ & $23(31.5)$ & $7(35.0)$ & $63(12.7)$ & \\
\hline Derived Japanese EQ-5D utility score, mean (SD) & $0.89(0.16)$ & $0.86(0.18)$ & $0.82(0.19)$ & $0.72(0.23)$ & $0.86(0.18)$ & $<0.001^{\mathrm{a}}$ \\
\hline EQ-5D overall VAS score, mean (SD) & $73.8(17.9)$ & $72.5(16.9)$ & $68.8(18.4)$ & $60.8(22.6)$ & $72.1(18.2)$ & $<0.001^{\mathrm{a}}$ \\
\hline \multicolumn{7}{|l|}{ WPAI score, mean (SD) } \\
\hline$\%$ Work time missed & $4.5(16.1)$ & $4.4(13.3)$ & $2.8(9.7)$ & $8.5(18.6)$ & $4.4(14.6)$ & $0.562^{\mathrm{a}}$ \\
\hline$\%$ Impairment at work & $29.5(25.5)$ & $35.8(27.6)$ & $37.3(29.4)$ & $42.2(25.1)$ & $33.0(26.9)$ & $0.051^{\mathrm{a}}$ \\
\hline$\%$ Overall work impairment & $30.5(26.7)$ & $37.0(28.9)$ & $38.1(29.4)$ & $44.7(28.0)$ & $34.2(28.0)$ & $0.058^{\mathrm{a}}$ \\
\hline$\%$ Activity impairment & $32.4(28.0)$ & $40.1(28.0)$ & $46.1(28.8)$ & $55.0(28.0)$ & $37.7(28.8)$ & $<0.001^{\mathrm{a}}$ \\
\hline
\end{tabular}

Continuous variables are reported as mean (SD) for non-missing observations; percentages are calculated as proportion of non-missing data. MIDAS score 0-5 = little or no disability; MIDAS score 6-10= mild disability; MIDAS score 11-20 = moderate disability; MIDAS score > 21 = severe disability; higher EQ-5D utility and VAS scores indicate higher general health status and quality of life, respectively

EQ-5D, EuroQol-5 Dimensions; HDM, headache days per month; MIDAS, Migraine Disability Assessment; SD, standard deviation; VAS, visual analog scale; WPAl, Work Productivity and Activity Impairment

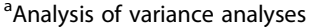

${ }^{\text {b}}$ Fisher's exact test 
with the findings of the American Migraine Prevalence and Prevention (AMPP) study (a postal-based, longitudinal survey of respondents with headache) $(94.5 \%$ and 5.5\% [18]) and the International Burden of Migraine Study (IBMS) (an internet-based, cross-sectional multinational survey) (94.3\% and 5.7\% [4]); however, the US Adelphi Migraine DSP included a greater proportion of patients with CM (90.8\% vs. 9.2\% [13]).

In the current study, no significant difference in employment status was observed between patients with EM and those with CM. This contrasts with the AMPP study and the US Adelphi Migraine DSP survey, both of which found significantly lower levels of full-time employment in people with CM than in those with EM $[13,18]$. Ford et al. also reported a significantly higher rate of unemployment among patients with CM than among those with EM.

Consistent with other studies [4, 13, 18, 19], women comprised three-quarters of the EM and CM cohorts in the current study. In Japan's aging society, the expectation that women will contribute to society as workers is high. Findings in other studies $[13,18]$ that patients with $\mathrm{CM}$ are less likely to be employed (full or part time) than those with EM may suggest that severe migraine could pose an obstacle for an ambitious woman in Japan. However, migraine severity did not impact significantly on full-time employment status in the current study, which probably reflects the Japanese belief that working hard is a virtue (gaman in Japanese, which is generally translated as 'perseverance', 'patience,' 'tolerance', or 'self-denial').

In common with the US Adelphi Migraine DSP [13], pain-related symptoms were the most frequent physician-reported migraine-related symptoms considered most troublesome to patients with EM or CM. However, apart from unilateral pain (seen in 51\% and $38 \%$ of patients with EM and CM, respectively, in the Japanese Adelphi Migraine DSP and 40\% and 43\%, respectively, in the US Adelphi Migraine DSP), frequencies were notably lower in Japanese patients than in US patients. For example, pulsating/throbbing pain was reported in $21 \%$ and $38 \%$ of patients with EM and CM in the Japanese Adelphi Migraine DSP, compared with $52 \%$ and $\sim 53 \%$ of patients in the US Adelphi Migraine DSP [13]. In migraine-related clinical trials, patients are usually asked to identify their most bothersome symptom (photophobia, phonophobia, or nausea) in addition to pain. In the US Adelphi Migraine DSP, photophobia as a physician-reported migraine-related symptom considered most troublesome to patients was reported for $18 \%$ and $26 \%$ of patients with EM and CM, respectively; phonophobia was reported for $8 \%$ and $19 \%$, respectively [13]; however, the prevalence of these symptoms was again much lower in patients in the Japanese Adelphi
Migraine DSP (photophobia: 1.5\% and 3.8\%, respectively, and phonophobia: $0.9 \%$ and $0 \%$, respectively). The concept of gaman may, again, have contributed towards these discrepant findings between patients with migraine in Japan and the USA, with concepts such as 'tolerance' and 'self-denial' resulting in Japanese patients being less likely to report migraine-related symptoms.

Comorbidity profiles varied between patients with EM and $\mathrm{CM}$ in Japan but, in contrast to other studies, not significantly. Anxiety and respiratory disorders were more common in patients with EM, and gastrointestinal problems and sleep disorders were more common in those with $\mathrm{CM}$, as was depression, a known risk factor for CM [20]. In the AMPP study, people with CM were twice as likely as those with EM to be depressed (odds ratio $[\mathrm{OR}] 2.0 ; 95 \%$ confidence interval $[\mathrm{CI}]$ 1.67-2.40; $p \leq 0.001$ ) or experience anxiety (OR 1.8 ; $95 \%$ CI $1.51-$ $2.15 ; p \leq 0.001)$. Similar findings were reported in the US Adelphi Migraine DSP [13]. The IBMS also reported higher rates of psychiatric disorders in patients with $\mathrm{CM}$ than in those with EM [4]. Respiratory disorders were reported to be more common in respondents with $\mathrm{CM}$ than in those with EM in the AMPP study [18].

Higher rates of cardiovascular risk factors (e.g., hypertension, high cholesterol, obesity) have also been reported in patients with CM than in those with EM $[4,13,18]$, and obesity is known to be an important modifiable risk factor for CM [20]. Although rates in the current study did not differ between EM and CM cohorts, hypertension and hyperlipidemia were seen in notable percentages $(\sim$ $28 \%$ and $\sim 20 \%$, respectively) of patients with EM or CM with comorbidities, and $\sim 10 \%$ of patients were obese. Correspondingly, $21-29 \%$ of patients were being prescribed antihypertensives/vasodilators, and $17-21 \%$ were receiving lipid-lowering agents for the treatment of comorbidities. Similar rates of hypertension and hyperlipidemia as comorbidities were also reported in patients with CM and EM in the US Adelphi Migraine DSP, but the rate of obesity was significantly higher in those with CM [13].

Although CM has been found in other studies to be associated with a greater societal economic burden than $\mathrm{EM}$, to our knowledge, this is the first study to note that healthcare resource use did not differ notably between patients with EM or CM in Japan. In the US Adelphi Migraine DSP study, people with CM incurred significantly greater rates of physician visits, migraine-related hospitalizations, and surgical interventions than those with EM [13]. Physician visits in the previous 12 months were significantly higher for those with $\mathrm{CM}$ consulting with neurologists in the USA [13]. Similar findings were also reported in the AMPP and IBMS studies [4, 21].

Physician-reported prescriptions for acute treatment in patients with EM or CM, mainly comprising triptans and NSAIDs, were generally in line with JSN/JHS 
guidelines [11]. Loxoprofen was the most widely used NSAID for the acute treatment of patients with migraine; in contrast, ibuprofen and naproxen appeared to be more widely used for this purpose in the US Adelphi Migraine DSP survey [13]. In the current study, opioid use as acute therapy was low in both patients with EM and those with CM $(0-0.2 \%)$, which again contrasts with the US Adelphi Migraine DSP survey findings (EM 8.3\% vs. CM $13.9 \%$; $p<0.001$ ) [13]. However, both the Japanese and the US Adelphi Migraine DSP surveys found that patients with $\mathrm{CM}$ were significantly more likely to experience $\mathrm{MOH}$ than were those with EM. In the Japanese Adelphi Migraine DSP, MOH was reported by physicians to be a problem with acute treatment in significantly more patients with CM than with EM $(p<$ 0.001).

Preventive therapy prescriptions were also generally in line with JSN/JHS guidelines [11]. It should be noted, however, that botulinum toxin and topiramate, which are used widely worldwide as preventive therapies for migraine (e.g., USA [13]), are approved in Japan for other diseases but not for migraine. Consequently, Japanese physicians have fewer treatment options for migraine prevention than physicians in other countries.

In the current study, nearly $60 \%$ and over $30 \%$ of patients with EM or CM, respectively, had never received a prescription for preventive therapy. While the percentage for patients with EM is similar to that reported in the US Adelphi Migraine DSP (50\%), Japanese patients with $\mathrm{CM}$ were almost twice as likely never to have received a prescription for preventive therapy as the corresponding percentage reported in the US DSP (17\%) [13]. Higher rates have been reported in population-based studies. For example, in the IBMS-II study, the percentage of patients with CM not currently using preventive therapy was 55\% [19], and in the AMPP study, only $33.3 \%$ of patients with $\mathrm{CM}$ reported that they were current users of preventive therapy [22]. Although the percentages from population-based studies are based on patient self-reports, they nevertheless suggest that a large number of patients with $\mathrm{CM}$ are not receiving the long-term preventive therapy that patients with severe and/or frequent migraines require [23]. The findings in the current study that over half of the patients with $\mathrm{CM}$ who were not receiving preventive therapy were consulting an internist rather than a neurologist could possibly indicate that non-specialist physicians or patients were reluctant to initiate such therapy. For example, patients and/or physicians may have concerns over the potential for adverse events with such long-term treatment [24, 25]. These findings may therefore highlight a need for ways of increasing the willingness of patients to accept and for physicians to initiate pharmacological migraine-preventive therapy.
According to physician-reported data, notable proportions of patients with EM or CM experienced at least one problem with their current acute $(>50 \%)$ or preventive ( $>20 \%$ ) therapy. Lack of efficacy was the mostly frequently identified problem with both acute and preventive treatment, occurring in over one-third of patients. This finding that many patients with migraine experienced problems with their therapy could be reflected in the number of prescriptions for second-, third-, or even fourth- (or higher)-line acute or preventive therapy observed in patients with EM or CM (although it should be borne in mind that the number of patients who received more than one line of therapy could have been influenced by the inclusion of a $10 \%$ oversampling of those who had failed at least one prior preventive treatment). In the previously mentioned AMPP study, $>40 \%$ of people with EM were found to have at least one unmet need with their current acute treatment, which included satisfaction with therapy (assessed as lack of efficacy, tolerance, or overall satisfaction with the medication); people with one unmet need (OR 1.49, 95\% CI 1.22-1.82) or two or more unmet needs (OR 2.21, 95\% CI 1.77-2.76) were more likely than those with no unmet needs to have used triptans in the past 3 months [26]. Lack of efficacy, tolerability, or both have also been reported to result in poor adherence and persistence with preventive medication in people with EM or CM $[19,23,27]$. In the current study, significantly more patients with CM than with EM had switched or discontinued acute or preventive treatment, and twice as many of those with CM than with EM were receiving current prescriptions for both acute and preventive treatments, findings that possibly reflect higher levels of unmet treatment need in people with CM. However, it is possible that the inclusion of a $10 \%$ oversampling of those who had failed at least one prior line of preventive treatment may have influenced this finding since the CM group was over-represented by these patients ( $22 \%$ vs. $10 \%$ of the EM group).

Headaches occurring in people with migraine have a substantial negative effect on the general health, HRQoL, and functioning of those affected [3, 4]. Our findings are in agreement with those of the US Adelphi Migraine DSP [13] and reveal a general trend for increased impairments in the patient-reported outcomes of headache-related disability, activity impairment, work productivity, and function as the number of HDM increased, with levels in the EM 8-14 HDM subgroup approaching or equaling those in the $\mathrm{CM} \geq 15 \mathrm{HDM}$ subgroup. For example, patients in the both EM 4-7 HDM and EM 8-14 HDM subgroups, as well as those in the $\mathrm{CM} \geq 15 \mathrm{HDM}$ subgroup, were impaired at work for $\sim 30 \%$ of the time. In contrast, the lack of a trend for an increase in WPAI scores for work time missed across 
HDM subgroups could again attest to the importance of gaman in Japanese culture. Such a finding could impact economic assessments of migraine on work productivity in Japan.

Impairments in general health status and HRQoL also increased with the frequency of HDM. Other studies $[13,28]$ have reported similar findings.

\section{Strengths/limitations}

One strength of this study has been the use of real-world data collected using a standardized methodology (as part of the Adelphi Migraine DSP), thus allowing direct comparison of migraine treatment patterns and disease burdens between Japan and other countries. Adelphi DSPs are large, multinational, cross-sectional observational studies of clinical practice providing valuable data on a range of common chronic diseases to supplement the findings of larger population-based studies [12]. As both clinical and patient-reported data are collected, we consider the Adelphi Migraine DSP to be an appropriate database for answering the research questions posed in this study. DSP data have previously helped elucidate treatment patterns in people with migraine in the USA, Germany, France, and Japan [13, 29-33].

All patient participants in the current study had been diagnosed with migraine by a physician; hence, the study findings should be considered generalizable to the consulting population of patients with EM or CM in Japan. However, generalizability is limited by the fact that study sites were selected based on the volume of patients with migraine routinely seen and, hence, consulting physicians were experienced in treating migraine.

Other limitations of the study include that the data are cross-sectional in nature; however, PRF data were considered suitable for the assessment of treatment patterns given that they were supplied by the physician and patient records could be referred to for patient history. Additionally, the Japanese Adelphi Migraine DSP was conducted over a 4-month period and included a limited number of physician and patient participants, especially patients with $\mathrm{CM}$. This may account for the lack of many significant differences between the EM and CM study populations that have been identified in population-based studies involving larger migraine populations (e.g., the AMPP and IBMS studies). There is also a potential for selection bias associated with the oversampling of patient participants who had failed at least one prior line of preventive therapy.

\section{Conclusions}

Managing migraine is challenging for both patients and physicians. As a guide for healthcare professionals, this analysis of the Japanese Adelphi Migraine DSP data provides a real-world snapshot of information on the clinical characteristics, disease burden, and treatment patterns of people with EM and CM being treated in clinical practice in Japan in 2014. In Japan, preventive treatment patterns differ in people with EM compared with those with CM, and both EM and CM pose notable disease burdens. Our data suggest that many patients were not receiving appropriate preventive therapy. These findings, together with the complexity of migraine, the heavy economic burden the disease places on healthcare resources, and the impact on people's ability to function, suggest an unmet need for more effective migraine therapies to reduce the burden of this disease. Importantly, further studies/research are needed to explore factors affecting patients' willingness to take preventive treatments and improve long-term outcomes.

\section{Additional files}

Additional file 1: Table S1. Drug classes being taken for the treatment of comorbidities by $\geq 5 \%$ of patients with episodic and chronic migraine and any comorbidity: physician-reported data. (DOCX 15 kb)

Additional file 2: Figure S1. Comorbid medical conditions reported in $>5 \%$ of patients with episodic or chronic migraine with any comorbid medical condition: physician-reported data provided for all patients with comorbidities (EM N = 455; CM N=29). Gl, gastrointestinal; COPD, chronic obstructive pulmonary disease. (TIF $1921 \mathrm{~kb}$ )

Additional file 3: Figure S2. Number of acute and prophylactic treatment lines ever received in patients with episodic migraine (EM) or chronic migraine (CM): physician-reported data. Lines of treatment could

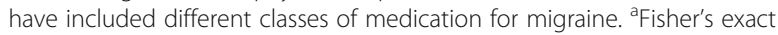
test. (TIF $1956 \mathrm{~kb}$ )

\section{Abbreviations}

AMPP: American migraine prevalence and prevention; ANOVA: Analysis of variance; Cl: Confidence interval; CM: Chronic migraine; DSP: Disease-Specific Programme; EM: Episodic migraine; EQ-5D: EuroQol-5 dimensions; HDM: Headache days per month; HRQoL: Health-related quality of life; IBMS: International burden of migraine study; IHS: International headache society; JHS: Japanese headache society; JSN: Japanese society of neurology; MIDAS: Migraine disability assessment; $\mathrm{MOH}$ : Medication-overuse headache; NSAID: Nonsteroidal anti-inflammatory drug; OR: Odds ratio; PRF: Patient record form; PSC: Patient self-completion; SD: Standard deviation; VAS: Visual analog scale; WPAI: Work Productivity and Activity Impairment; YLD: years lived with disability

\section{Acknowledgements \\ The authors would like to acknowledge Gill Gummer and Caroline Spencer (Rx Communications, Mold, UK) for medical writing assistance with the preparation of this manuscript, funded by Eli Lilly and Company. \\ Funding \\ This study was funded by Eli Lilly and Company. \\ Availability of data and materials \\ The data that support the findings of this study are available from Adelphi Real World, but restrictions apply to the availability of these data, which were used under license for the current study and so are not publicly available. However, data are available from the authors upon reasonable request and with permission from Adelphi Real World.}

\section{Authors' contributions}

All named authors meet the International Committee of Medical Journal Editors (ICMJE) criteria for authorship of this article, have contributed sufficiently to the work and provided critical revision of the manuscript for 
important intellectual content. All authors had full access to all of the data in this study and take complete responsibility for the integrity of the data and accuracy of the data analysis. All authors have given their approval for this version to be published in The Journal of Headache and Pain.

\section{Ethics approval and consent to participate}

This study was conducted in accordance with the ethical principles that have their origin in the Declaration of Helsinki and that are consistent with Good Pharmacoepidemiology Practices and the applicable laws and regulations of Japan. As this was a retrospective analysis that used deidentified data that had been previously collected, patient participants were not required to provide formal Consent to Release Information forms for the current analyses; the original consent from those who provided data in the DSP covered the planned analyses in this study.

\section{Consent for publication}

Not applicable.

\section{Competing interests}

KU, WY, LL, AK, YK, and TT are full-time employees of Eli Lilly and Company. SC and JJ are full-time employees of Adelphi Real World.

\section{Publisher's Note}

Springer Nature remains neutral with regard to jurisdictional claims in published maps and institutional affiliations.

\section{Author details}

${ }^{1}$ Eli Lilly Japan K.K., 5-1-28, Isogamidori, chuo-ku, Kobe-shi 651-0086, Japan. Eli Lilly and Company, Lilly Corporate Center, Indianapolis, IN 46285, USA. ${ }^{3}$ Adelphi Mill, Grimshaw Lane, Bollington, Cheshire SK10 5JB, UK. ${ }^{4}$ Lilly Hungária Kft, Madách Imre út 14, Budapest 1075, Hungary.

Received: 14 February 2019 Accepted: 5 May 2019

Published online: 07 June 2019

\section{References}

1. ICHD-3. Headache Classification Committee of the International Headache Society (IHS). The International Classification of Headache Disorders, 3rd edition. Cephalalgia. 2018;38:1-211. https://www.ichd-3.org/. Accessed 22 Nov 2018

2. Katsarava Z, Buse DC, Manack AN (2012) Defining the differences between episodic migraine and chronic migraine. Curr Pain Headache Rep 16:86-92

3. Lipton RB, Hamelsky SW, Kolodner KB, Steiner TF, Stewart WF (2001) Migraine, quality of life, and depression: a population-based case-control study. Neurology. 55(5):629-635

4. Blumenfeld AM, Varon SF, Wilcox TK, Buse DC, Kawata AK, Manack A et al (2010) Disability, HRQoL and resource use among chronic and episodic migraineurs: results from the international burden of migraine study (IBMS). Cephalalgia. 31(3):301-315

5. GBD 2016 Disease and Injury Incidence and Prevalence Collaborators (2017) Global, regional, and national incidence, prevalence, and years lived with disability for 328 diseases and injuries for 195 countries, 1990-2016: a systematic analysis for the Global Burden of Disease Study 2016. Lancet 390:1211-1259

6. Woldeamanuel YW, Cowan RP (2017) Migraine affects 1 in 10 people worldwide featuring recent rise: a systematic review and meta-analysis of community-based studies involving 6 million participants. J Neurol Sci 372: 307-315

7. Sakai F, Igarashi H (1997) Prevalence of migraine in Japan: a nationwide survey. Cephalalgia. 17(1):15-22

8. Takeshima T, Ishizaki K, Fukuhara Y, Ijiri T, Kusumi M, Wakutani Y et al (2004) Population-based door-to-door survey of migraine in Japan: the Daisen study. Headache. 44(1):8-19

9. Suzuki S, Hirata K, Tatsumoto M, Hoshiyama E, Kobayashi H (2005) The prevalence and character of primary headache in Japanese high school students. Rinsho Shinkeigaku 45(10):717-723

10. Ando N, Fujimoto S, Ishikawa T, Teramoto J, Kobayashi S, Hattori A et al (2007) Prevalence and features of migraine in Japanese junior high school students aged 12-15 yr. Brain and Development 29(8):482-485

11. Japanese Society of Neurology and the Japanese Headache Society. Clinical practice guideline for chronic Headache 2013. http://www.jhsnet.org/ english/guideline2013.pdf
12. Anderson P, Benford M, Harris N, Karavali M, Piercy J (2008) Real-world physician and patient behaviour across countries: disease-specific programmes - a means to understand. Curr Med Res Opin 24(11):3063-3072

13. Ford JH, Jackson J, Milligan G, Cotton S, Ahl J, Aurora S (2017) A real-world analysis of migraine: a cross-sectional study of disease burden and treatment patterns. Headache. 57(10):1532-1544

14. Stewart WF, Lipton RB, Kolodner K, Liberman J, Sawyer J (1999) Reliability of the migraine disability assessment score in a population-based sample of headache sufferers. Cephalalgia. 19(2):107-114

15. Stewart WF, Lipton RB, Dowson AJ, Sawyer J (2001) Development and testing of the migraine disability assessment (MIDAS) questionnaire to assess headache-related disability. Neurology. 56(6 Suppl 1):S20-\$28

16. Schrag A, Selai C, Jahanshahi QNP (2000) The EQ-5D - a generic quality of life measure - is a useful instrument to measure quality of life in patients with Parkinson's disease. J Neurol Neurosurg Psychiatry 69:67-73

17. Reilly MC, Zbrozek AS, Dukes EM (1993) The validity and reproducibility of a work productivity and activity impairment instrument. Pharmacoeconomics. 4(5):353-365

18. Buse DC, Manack A, Serrano D, Turkel C, Lipton RB (2010) Sociodemographic and comorbidity profiles of chronic migraine and episodic migraine sufferers. J Neurol Neurosurg Psychiatry 81:428-432

19. Blumenfeld AM, Bloudek LM, Becker WJ, Buse DC, Varon SF, Maglinte GA et al (2013) Patterns of use and reasons for discontinuation of prophylactic medications for episodic migraine and chronic migraine: results from the second international burden of migraine study (IBMS-II). Headache. 53(4): 644-655. https://doi.org/10.1111/head.12055

20. May A, Schulte LH (2016) Chronic migraine: risk factors, mechanisms and treatment. Nat Rev Neurol 12(8):455-464

21. Munakata J, Hazard E, Serrano D, Klingman D, Rupnow MF, Tierce J et al (2009) Economic burden of transformed migraine: results from the American Migraine Prevalence and Prevention (AMPP) study. Headache. 49:498-508

22. Bigal ME, Serrano D, Reed M, Lipton RB (2008) Chronic migraine in the population. Burden, diagnosis, and satisfaction with treatment. Neurology. 71:559-566

23. D'Amico D, Tepper SJ (2008) Prophylaxis of migraine: general principles and patient acceptance. Neuropsychiatr Dis Treat 4(6):1155-1167

24. Dekker F, Knuistingh Neven A, Andriesse B, Kernick D, Reis R, Ferrari MD et al (2012) Prophylactic treatment of migraine; the patient's view, a qualitative study. BMC Fam Pract 13:13

25. Dekker F, Neven AK, Andriesse B, Kernick D, Ferrari MD, Assendelft WJ (2012) Prophylactic treatment of migraine by GPs: a qualitative study. $\mathrm{Br} J$ Gen Pract 62(597):e268-e274

26. Lipton RB, Buse DC, Serrano D, Holland S, Reed ML (2013) Examination of unmet treatment needs among persons with episodic migraine: results of the American Migraine Prevalence and Prevention (AMPP) study. Headache. 53:1300-1311

27. Hepp Z, Bloudek LM, Varon SF (2014) Systematic review of migraine prophylaxis adherence and persistence. J Manage Care Pharm 20:22-33

28. Torres-Ferrus M, Quintana M, Fernandez-Morales J, Alvarez-Sabin J, PozoRosich P (2017) When does chronic migraine strike? A clinical comparison of migraine according to the headache days suffered per month. Cephalalgia. 37:104-113

29. Mutebi A, Pike J, Shah N, Jackson J, Cotton S, Desai PR et al (2016) The use of migraine prophylaxis treatment: analysis of clinical practice data from the United States, Germany, France, and Japan. Value Health 19(3):A68

30. Mutebi A, Pike J, Shah N, Jackson J, Cotton S, Desai P, et al. Unmet need in migraine prophylaxis treatment in the United States of America: data from clinical practice. Abstract presented at the $58^{\text {th }}$ Annual Scientific Meeting of the American Headache Society; San Diego, CA; 2016 (PS46)

31. Pike J, Mutebi A, Shah N, Jackson J, Cotton S, Desai PR et al (2016) Factors associated with a history of failure and switching migraine prophylaxis treatment: an analysis of clinical practice data from the United States, Germany, France and Japan. Value Health 19(3):A68

32. Pike J, Mutebi A, Shah N, Jackson J, Cotton S, Desai PR, et al. Migraine prophylaxis treatment patterns in Japan: analysis of data from clinical practice (Adelphi DSP 2014). Poster presented at Japan headache society congress; Kyoto; 21-22 Oct 2016; poster 017-4

33. Shah N, Pike J, Mutebi A, Jackson J, Cotton S, Desai P, et al. The use of migraine prophylaxis treatments in the United States of America: analysis of data from clinical practice. Abstract presented at the $58^{\text {th }}$ Annual Scientific Meeting of the American Headache Society; San Diego, CA; 9-12 Jun 2016 (PS45) 